\title{
Increased glutamate and deep brain atrophy can predict the severity of multiple sclerosis
}

\author{
Hubert Polacek ${ }^{a}$, Ema Kantorova ${ }^{b}$, Petra Hnilicovac, Marian Grendar ${ }^{c}, K^{c}$ amil Zelenak ${ }^{d}$, Egon Kurca ${ }^{b}$
}

\begin{abstract}
Objective. In multiple sclerosis (MS), deep grey matter (DGM) atrophy has been recognised as a crucial component of the disease that presents early and it has been associated with disability. Although the precise mechanism underlying grey matter atrophy is unknown, several hypotheses have been postulated. Our previous research pointed to correlations of hypothalamic metabolic alterations with clinical outcomes of MS, therefore we decided to further test the relationship of these alterations with DGM atrophy.

Methods. We used ${ }^{~} \mathrm{H}$-Magnetic Resonance spectroscopy ('H-MRS) of the hypothalamus to test its metabolites in 26 patients with RRMS and 22 healthy age-matched controls. DGM atrophy was evaluated by simple planimetry of third ventricular width on the hypothalamic level ( $3 \mathrm{VW}$ ) in T1 weighted MRI pictures. Metabolite ratios of $\mathrm{N}$-acetyl aspartate (NAA), choline (Cho), glutamate and glutamine (Glx), myo-inositol (mlns) and creatine ( $\mathrm{Cr}$ ) were correlated with Multiple Sclerosis Severity Scale (MSSS) and 3VW.

Results. Metabolite concentrations were compared between patients and controls using multiple regression models allowing for age, $3 \mathrm{VW}$ and metabolites. It revealed that the only relevant predictors of MSSS were 3VW and Glx/NAA. At a significance level of $P<0.05$, a unit increase of $3 \mathrm{VW}$ was associated with a 0.35 increase of MSSS, for a typical value of GIx/NAA; $P$ value 0.0039 . A unit increase of Glx/NAA was associated with a 0.93 increase of MSSS, for a typical value of atrophy; $P$ value 0.090 . There were significant linear correlations between GIx/Cr and MSSS, GIx/NAA and MSSS, and between mIns/NAA and 3VW.

Conclusions. The results suggest that both NAA and GIx are associated with neurodegeneration of hypothalamic DGM and severe disease course. Glx related ${ }^{1} \mathrm{H}-\mathrm{MRS}$ parameters seem to be superior to other metabolites in determining disease burden, independently of otherwise powerful 3VW planimetry. Significantly increased mlns/NAA in MS patients compared to controls point to gliosis, which parallels the atrophy of hypothalamic DGM.
\end{abstract}

Key words: multiple sclerosis, predictors, disease severity, neurodegeneration, ${ }^{1} \mathrm{H}-\mathrm{MRS}$, brain metabolites, width of the third ventricle, brain atrophy

Received: February 10, 2018; Accepted: June 19, 2018; Available online: July 16, 2018

https://doi.org/10.5507/bp.2018.036

${ }^{a}$ Clinic of Nuclear Medicine, Jessenius Faculty of Medicine in Martin, Comenius University

Bratislava, Slovak Republic

${ }^{b}$ Clinic of Neurology, Jessenius Faculty of Medicine in Martin, Comenius University Bratislava, Slovak Republic

'Biomedical Centre, Jessenius Faculty of Medicine in Martin, Comenius University Bratislava, Slovak Republic

${ }^{d}$ Clinic of Radiodiagnostics, Jessenius Faculty of Medicine in Martin, Comenius University Bratislava, Slovak Republic

Corresponding author: Ema Kantorova, e-mail: kantorova@jfmed.uniba.sk

\section{INTRODUCTION}

Multiple sclerosis (MS) is a heterogeneous disorder with almost unpredictable disease course. Although the generally accepted opinion regards MS as primarily autoimmune disease, there is a growing body of evidence that disability is not clearly related to inflammatory lesions but rather to a progressive and diffuse neuro-axonal loss ${ }^{1}$. Deep grey matter (DGM) may be affected early $y^{2-4}$ and its damage may determine the course of MS (ref. $\left.{ }^{5,6}\right)$. Despite the widely accepted neurodegenerative hypothesis of MS, the mechanisms of degeneration are not yet sufficiently understood.

In the presented pilot study we focused on the hypothalamus (HYP) - a crucial central structure in the subcortical neuro-endocrino-immunological circuits maintaining the body homeostasis. Several previous postmortem studies revealed early and severe damage of HYP in MS (ref. ${ }^{3,4}$ ). To evaluate both inflammatory and neurodegenerative changes in this brain region non-invasively, we measured HYP metabolic changes using ${ }^{1} \mathrm{H}-\mathrm{MRS}$ in similar way to previous studies, analysing other brain regions $^{7-11}$.

${ }^{1} \mathrm{H}-\mathrm{MRS}$ parameters of three metabolites have been reported to reliably evaluate the three main aspects of neurodegeneration:

$\mathrm{N}$-acetylaspartate (NAA) (ref. ${ }^{7,9}$ ) is an amino acid which is detected almost exclusively in neurons. NAA decline is interpreted as being caused by loss of neurons and their connections, characteristic for chronic lesions and atrophic grey matter structures ${ }^{8,9,12}$. Energetically favourable NAA can serve as a reservoir of glutamate (Glu) $\left(\right.$ ref. $\left.^{13}\right)$.

Glutamate (the main part of glutamate and glutamine complex) is the main excitatory neurotransmitter playing an essential role in development and the neuroplasticity 
of the CNS (ref. ${ }^{14}$ ). Excessively increased Glu concentrations are responsible for neuronal loss in both acute and chronic stages of neurodegenerative disorders ${ }^{15}$. Recent evidence points to the crucial role of Glu in the pathogenesis of MS (ref. $\left.{ }^{10,16,17}\right)$.

Myo-Inositol (mIns) (ref. ${ }^{7,18}$ ) is a marker of glial cellularity ${ }^{18}$. Increased levels of mIns indicate increased number of glial cells (gliosis) typical for both inflammatory MS lesions and normal appearing brain tissue $e^{8,11,17,19}$ and may precede NAA reduction in MS $\left(\right.$ ref. ${ }^{20}$ ).

\section{Other ${ }^{1} \mathbf{H}$-MRS metabolites}

Choline (Cho) (ref. ${ }^{7,8}$ ) concentration mainly indicates the amount of membrane phospholipids, which can be increased during active myelin breakdown or turnover, characteristic for active MS demyelinating lesions ${ }^{8}$. On the other hand, in atrophic DGM, concentration of Cho may remain intact ${ }^{12}$.

Creatine $(\mathrm{Cr})\left(\right.$ ref. $\left.^{7-9}\right)$ complex, composed of creatine and phosphocreatine, is a good indicator of cellular (both glial and neuronal) energy metabolism. Since the absolute $\mathrm{Cr}$ concentrations have been found stable throughout all central nervous system, they are often used as an internal standard to evaluate all other previously mentioned metabolites. However, hypercellularity in active inflammation or gliosis can also increase $\mathrm{Cr}$ concentration, thus complicating interpretation of other commonly evaluated metabolites-to-creatine ratios ${ }^{8,9}$

To evaluate morphological impact of hypothalamic neurodegeneration, we used the modified planimetric parameter obtained from routine anatomical MRI-the width of the third ventricle ( $3 \mathrm{VW}$ ). Many previous studies confirmed the value of $3 \mathrm{VW}$ morphometry in assessment of subcortical DGM volume loss and its impact on clinical course of MS (ref., ${ }^{1,21-23}$ ).

The aim of this study was to evaluate the metabolic background of hypothalamic neurodegeneration and to test whether it correlates with disease burden and subcortical atrophy. We hypothesised that imaging changes of the HYP can distinguish MS patients from healthy individuals as confirmed for other brain structures in previous studies. We chose RRMS patients to test the hypothesis that neurodegeneration is a substantial hallmark of multiple sclerosis. Since it determines the progression of neurological disability ${ }^{5,6}$, we presuppose that neurodegeneration does not preclude earlier, relapsing forms of the disease.

\section{METHODS}

\section{Subjects}

26 adult patients with RRMS and 22 age- and sexmatched control participants were included in the study after giving their written consent. The protocol was approved by the ethics committee of Jessenius Faculty of Medicine, Comenius University (reference number 1678/2015).

Patients with clinically definite MS according to McDonald criteria 2005 (ref. ${ }^{24}$ ) were prospectively selected from the Multiple Sclerosis Centre, University Hospital in Martin, Slovak Republic. Four patients, in which neuromyelitis optica was not excluded by radiological/clinical examinations, were tested for the presence of anti-aquaporin- 4 antibodies with negative results. All patients were treated with immunomodulatory agents (interferon beta, glatiramer acetate, natalizumab, fingolimod) depending on their disease activity. The treatments were in accordance with national guidelines (www.health.gov). Within the last 12 months before ${ }^{1} \mathrm{H}-\mathrm{MRS}$ imaging ${ }^{7}$, patients had remained on the same treatment. Clinical disability was evaluated by neurologists specialised in MS by Expanded Disability Status Scale (EDSS) and additionally adjusted for disease duration by Multiple Sclerosis Severity Scale (MSSS) $\left(\right.$ ref. $\left.^{25}\right)$.We chose MSSS (ref. ${ }^{25}$ ) for correlations due to its better ability to reflect the speed of progression (severity) of MS (ref. ${ }^{25}$ ).

Control participants $(\mathrm{CON})$ were recruited from among volunteers (e.g. medical staff, students, partners of patients, or patients with other than brain disease -e.g. moderate lower back pain, carpal tunnel syndrome). No pregnancy, endocrine, oncological or other serious disorders, possibly leading to hypothalamic impairment, were confirmed in either patients or controls.

Demographic and clinical data of both patients and controls are summarised in Table 1.

Table 1. Demographic and clinical data of patients with multiple sclerosis and healthy controls.

\begin{tabular}{|c|c|c|c|}
\hline Patients & MS & $\mathrm{CON}$ & \\
\hline Number of subjects & 26 & 22 & \\
\hline Age (years) & $33.9(22-59)$ & $34.6(20-59)$ & ${ }^{*} P=0.59$ \\
\hline $\operatorname{Sex}(M / F)$ & $7 / 19$ & $8 / 14$ & \\
\hline Disease duration (years) & $7.7(2-22)$ & NA & \\
\hline EDSS & $2.6(1-6)$ & NA & \\
\hline MSSS & $4.2(1-8.1)$ & NA & \\
\hline
\end{tabular}

$\mathrm{MS}=$ patients with multiple sclerosis, $\mathrm{M}=$ male, $\mathrm{F}=$ female, $\mathrm{CON}=$ healthy controls, EDSS = Expanded Disability Status Scale, MSSS = Multiple Sclerosis Severity Scale, NA = not applicable, * Two sample T-test, statistical significance $\mathrm{P}=0.05$ 


\section{Imaging protocol}

Each subject underwent structural MRI scanning a few minutes before spectroscopy acquisition. Images were acquired using a 1.5 T Siemens Symphony scanner in the MRI unit of Radiology Clinic at the University Hospital in Martin, Slovak Republic. Structural MRI included T1weighted MP-RAGE, T2-weighted and FLAIR sequences.

Due to well-known issues of magnetic field $\left(\mathrm{B}_{0}\right)$ inhomogeneity, especially in marginal brain regions located close to sinuses, phase maps of $\mathrm{B}_{0}$ were obtained prior to ${ }^{1} \mathrm{H}$-MRS acquisition in each subject. The volume of interest for $\mathrm{B}_{0}$ homogeneity adjustments (shimming volume) was manually placed at HYP region outside any $B_{0}$ distortions visible on the $\mathrm{B}_{0}$ map. Maximum possible $\mathrm{B}_{0}$ homogeneity was then achieved by iterative field mapping and calculation of appropriate shim currents of the first- and second-order corrections. Three-dimensional spectroscopic imaging sequence based on Point Resolved Spectroscopy (PRESS) was used to acquire localised data focused on HYP region (TE/TR $=30 / 1500 \mathrm{~ms}$, FOV was $10 \times 10 \times 8$, interpolated to $16 \times 16 \times 8 \mathrm{~cm}^{3}$ with a nominal voxel size of $10 \times 10 \times 12.5 \mathrm{~mm}^{3}$ and volumes of interest (VOIs) $50 \times 60 \times 35 \mathrm{~mm}^{3}$, elliptical weighting, $7 \mathrm{~min}$ acquisition.

We preferred to use multi-voxel over single-voxel ${ }^{1} \mathrm{H}-\mathrm{MRS}$ due to its advantages of shorter measurement time for two voxels and especially due to voxel shifting in post-processing. During data acquisition, the signal from magnetically heterogeneous area under HYP was suppressed by a suppression slab.

\section{Evaluation of structural MRI and ${ }^{1} \mathrm{H}$-MRS spectra}

Structural MRI images of all subjects were inspected for macroscopic hypothalamic lesions by the radiologist blinded to participant's disease status. ${ }^{1} \mathrm{H}-\mathrm{MRS}$ ( ref. $^{7}$ ) data were evaluated by a blinded physicist. Spectra of two voxels, precisely located to the central part of the right and left hypothalamus in jSIPROsoftware ${ }^{26}$ were analysed using LCModel $^{26}$ software. The voxel centre was placed between anterior commissure and mammillary body $\sim 1 \mathrm{~mm}$ anteriorly to fornix in sagittal and $\sim 2 \mathrm{~mm}$ laterally to the border of $3^{\text {rd }}$ ventricle in trans-axial plane (Fig. 1A). Five main metabolites: Cr, NAA, Cho, Glx and mIns were quantified in each voxel spectra. If the quality of the spectra was too low (FWHM > $0.14 \mathrm{ppm}$ ) or the output from LCModel showed evident misinterpretation, we searched for a better signal strength by shifting both voxels backwards in anteroposterior direction (in 5 of 51 subjects). In 2 subjects, less than $5 \mathrm{~mm}$ voxel replacement solved the low quality issue and the new values were used for analysis. Remaining 3 subjects ( 1 patient and 2 controls) were excluded.

To circumvent complicated evaluation of partial volume effects of water on the peak amplitudes, clinically relevant metabolite ratios rather than individual metabolite concentrations were evaluated (Figure 1B). In addition to traditional NAA/Cr, Cho/Cr and NAA/Cho (ref. ${ }^{8,9}$ ) we also analysed Glx/Cr, Glx/NAA and mIns/NAA ratios, being encouraged by our previous experience ${ }^{27}$ and similar studies ${ }^{10,11,17}$. Since the values from both hypothalamic voxels were highly intercorrelated for all ratios in both patients and controls ( $r>0.5, P<0.0001)$, the whole hypothalamus values, calculated as the means from left and right side spectra, were used for statistical analyses.

\section{Linear (planimetric) measurement of subcortical brain atrophy -third ventricle width (3VW)}

To achieve the best reproducibility, measurements were performed using fully automated in-house made software with $0.1 \mathrm{~mm}$ linear resolution. In each subject, T1-weighed MP-RAGE dataset was reoriented to AC-PC plane and the trans-axial slice $2 \mathrm{~mm}$ below the anterior commissure was selected. After denoising, up-sampling, and segmentation of the images, $3 \mathrm{VW}$ was defined as the maximum transverse dimension of $3 \mathrm{VW}$ between tuberal regions of HYP (Fig. 1C).

\section{Statistical analyses}

Correlations between metabolites and predictors (age, 3VW, disease duration, EDSS, MSSS) were assessed by the Pearson correlation coefficients, with the $95 \%$ confidence intervals and $P$-values computed by the R library psychometric $^{28,29}$. Statistical assessment of a difference between correlations was obtained by the R library CoCor (ref. ${ }^{30}$ ). Differences between cases and controls in ratios of metabolites were evaluated by Student's independent two-sample t-test, after Shapiro Wilkinson test of normality and Levene's test of equality of variances. Classification into case/control status by the age, $3 \mathrm{VW}$ and metabolites was performed by the logistic regression in R library MASS ( ref. $^{31}$ ).

Table 2. Summary of ${ }^{1} \mathrm{H}-\mathrm{MRS}$ metabolite ratios in MS patients and healthy controls.

\begin{tabular}{llll}
\hline${ }^{1} \mathrm{H}-\mathrm{MRS}$ ratio & \multicolumn{1}{c}{$\mathrm{MS}$} & \multicolumn{1}{c}{$\mathrm{CON}$} & $\mathrm{n}=22$ \\
& $\mathrm{n}=26$ & $1.5(1.18-1.99)$ & 0.0002 \\
$\mathrm{NAA} / \mathrm{Cr}$ & $1.31(0.9-1.7)$ & $0.39(0.29-0.49)$ & 0.005 \\
$\mathrm{Cho} / \mathrm{Cr}$ & $0.35(0.26-0.48)$ & $2.3(1.04-4.08)$ & 0.35 \\
$\mathrm{Glx} / \mathrm{Cr}$ & $2.22(0.89-4.33)$ & $0.62(0.17-1.19)$ & 0.029 \\
mIns /NAA & $0.75(0.37-1.24)$ & $3.84(2.63-4.93)$ & 0.19 \\
NAA/Cho & $3.69(2.65-4.94)$ & $1.5(0.75-2.35)$ & 0.089 \\
Glx/NAA & $1.69(0.66-2.73)$ & & \\
\hline
\end{tabular}

HYP = hypothalamus, MS = patients with multiple sclerosis, $\mathrm{CON}=$ healthy controls, $\mathrm{Cho} / \mathrm{Cr}=\mathrm{choline}$ to creatine ratio, $\mathrm{NAA} / \mathrm{Cr}=\mathrm{N}$-acetylaspartate to creatine ratio, $\mathrm{mIns} / \mathrm{NAA}=$ myo-inositol to $\mathrm{N}$-acetyl-aspartate ratio, $\mathrm{Glx} / \mathrm{Cr}=$ glutamate and glutamine to creatine ratio, $\mathrm{Glx} / \mathrm{NAA}=$ glutamate and glutamine to $\mathrm{N}$-acetyl-aspartate ratio, NAA/Cho $=\mathrm{N}$-acetyl-aspartate to choline ratio, Two sample T- test, $\alpha=0.05$ 
A
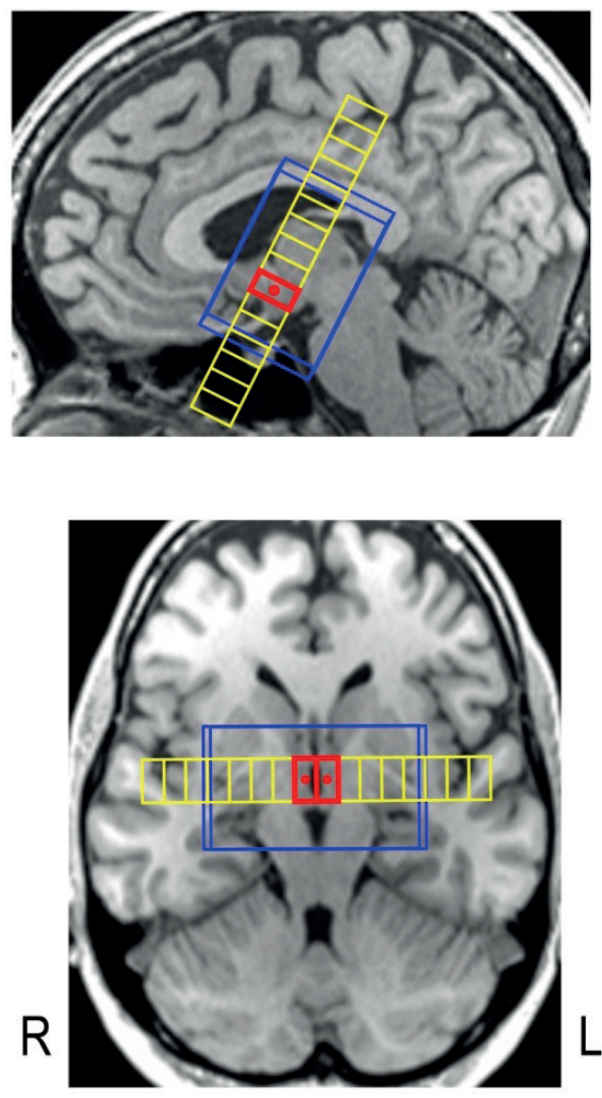

B

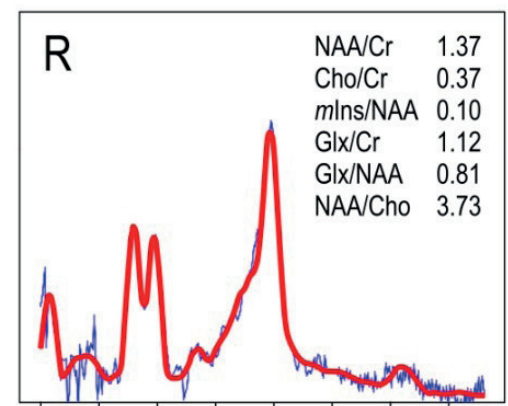

$\begin{array}{llllllll}4.0 & 3.5 & 3.0 & 2.5 & 2.0 & 1.5 & 1.0 & 0.5\end{array}$

ppm

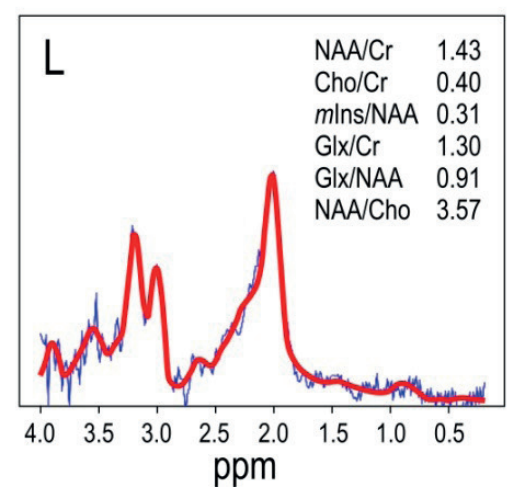

Patient with MS
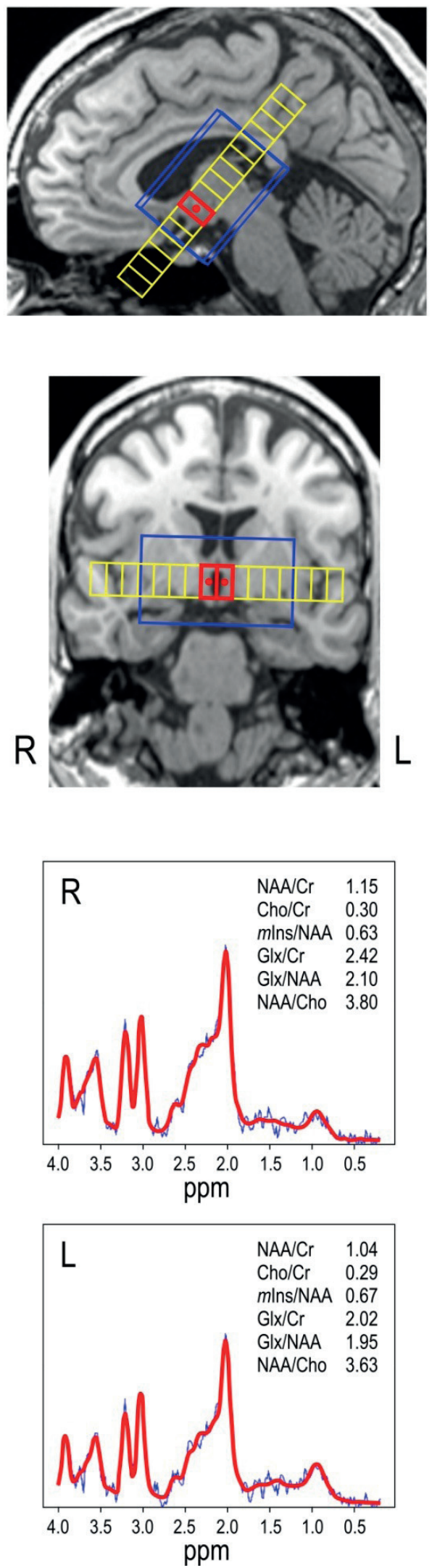

Fig. 1A,B. Morphological $T_{1}$-weighted reference images display the positioning of the targeting right (R) and left (L) hypothalamic ${ }^{1} \mathrm{H}$-MRS voxels (red squares) in the representative patient with multiple sclerosis and the control participant (A). Acquired matrix size was $10 \times 10 \times 8$, interpolated to $16 \times 16 \times 8$ (yellow squares) with a nominal voxel size of $10 \times 10 \times 12.5 \mathrm{~mm}^{3}$ and volumes of interest (VOIs) $50 \times 60 \times 35 \mathrm{~mm}^{3}$ (blue squares). LCModel fitted spectra (red curves) with relative levels of metabolite ratios corresponding to selected voxels are shown below (B). 

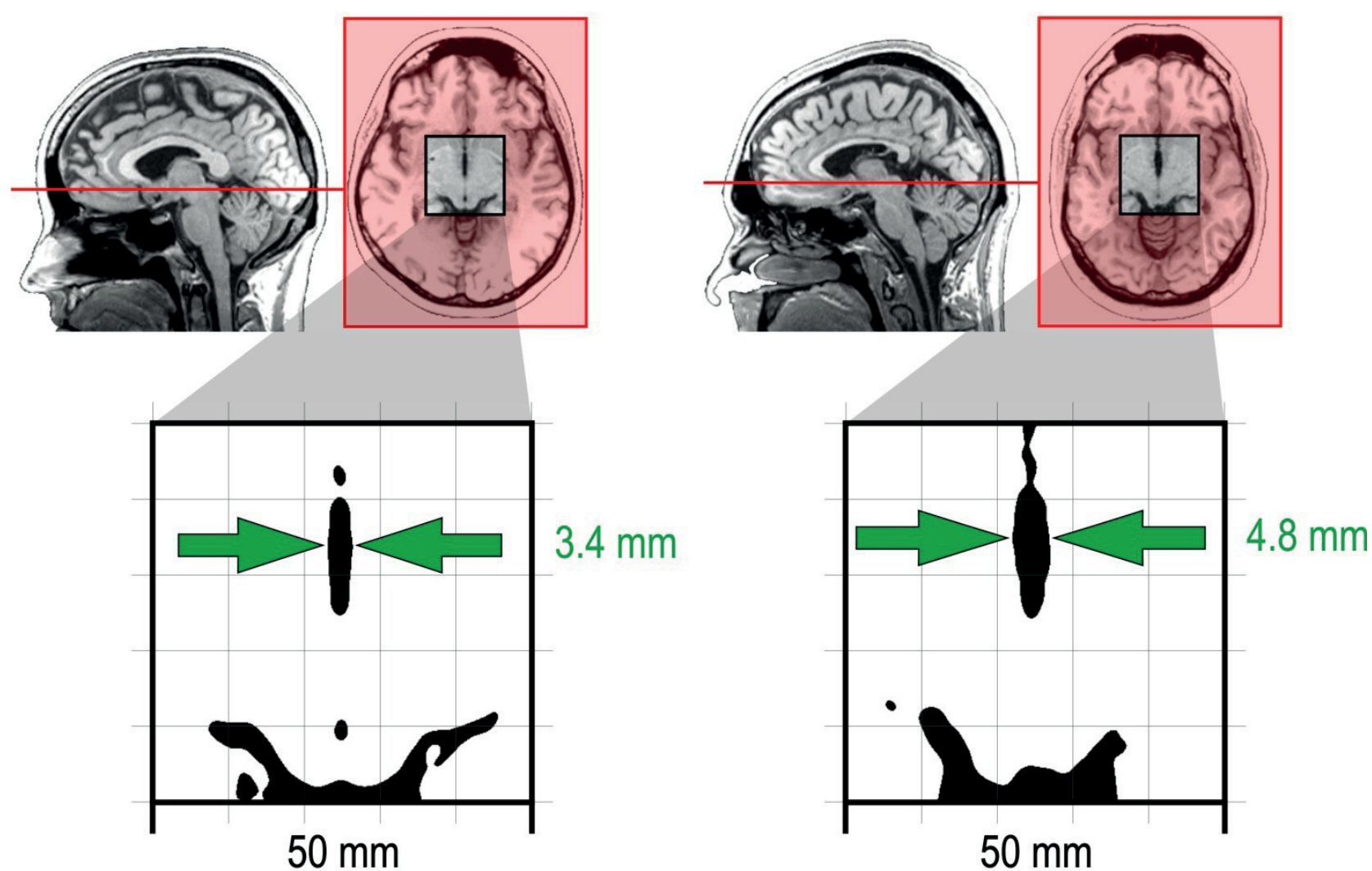

Fig. 1C. Measurements were performed using fully automated in-house software with $0.1 \mathrm{~mm}$ linear resolution. In each subject, T1-weighed MP-RAGE dataset was reoriented to AC-PC plane and the trans-axial slice $2 \mathrm{~mm}$ below the anterior commissure was selected. After denoising, up-sampling, and segmentation of the images, $3 \mathrm{VW}$ was defined as the maximum transverse dimension of $3 \mathrm{VW}$ between tuberal regions of HYP.

\section{RESULTS}

Our results are based on data from 26 RRMS patients and 22 controls. Exploratory data analysis showed significant differences between RRMS and CON groups in: NAA/Cr, Cho/Cr, and mIns/NAA, and to a lesser extent Glx/NAA ratio. In comparison with $\mathrm{CON}$, patients' $\mathrm{NAA} / \mathrm{Cr}$ and $\mathrm{Cho} / \mathrm{Cr}$ concentrations were decreased, and mIns/NAA and Glx/NAA increased. Evaluation of metabolic ratios is summarised in Table 2 .

The third ventricle was significantly wider in RRMS compared to CON $(4.2 \mathrm{~mm} \pm 1.5 \mathrm{~mm}$ SD versus $2.6 \mathrm{~mm}$ $\pm 0.99 \mathrm{~mm} \mathrm{SD}, P=0.0001$, Two sample T-test).

\section{Identification of relevant predictors}

Logistic regression after the AIC (ref. ${ }^{31}$ ) model selection found that age, $3 \mathrm{VW}, \mathrm{Glx} / \mathrm{Cr}, \mathrm{Glx} / \mathrm{NAA}$, and to a lesser extent NAA/Cr and NAA/Cho, can be important predictors of MS status. AUC associated with the model is $94.1 \%$.

Metabolite concentrations were compared between RRMS and CON using multiple (linear) regression models allowing for age, $3 \mathrm{VW}$ and metabolites. It revealed that the only relevant predictors of MSSS were 3VW and Glx/ NAA. At a significance level of $P<0.05$, a unit increase of $3 \mathrm{VW}$ was associated with a 0.35 increase of MSSS, for a typical value of Glx/NAA; $P$ value 0.0039 . A unit increase of Glx/NAA was associated with a 0.93 increase of MSSS, for a typical value of atrophy; $P$ value 0.090 . The data are given in Table 3.

Table 3. Identification of the most relevant predictors via regression modelling.

\begin{tabular}{lll}
\hline Predictors & MSSS & \multicolumn{1}{c}{$P$} \\
\hline 3VW & 0.3526 & 0.0039 \\
Glx/NAA & 0.9346 & 0.09 \\
\hline
\end{tabular}

$3 \mathrm{VW}=$ width of the third ventricle, Glx/NAA = glutamate and glutamine to N-acetyl-aspartate ratio, MSSS = Multiple Sclerosis Severity Scale, the Akaike Information Criterion model- AIC(ref. ${ }^{31}$ )

\section{Correlations of predictors and clinical markers}

Pearson correlations of MSSS score with tissue metabolite concentrations, 3VW, age and disease duration were significant for the following: positive correlation between Glx/Cr and MSSS; between Glx/NAA and MSSS; and between mIns/NAA and 3VW. NAA/Cr and Cho/ $\mathrm{Cr}$ did not correlate with any of the markers studied. The data are given in Table 4. 
Table 4. Correlations of the metabolite ratios with clinical parameters and 3VW in RRMS patients.

\begin{tabular}{lccll}
\hline Metabolite ratios & Age & DD & MSSS & 3VW \\
\hline NAA/Cr & ns & ns & ns & ns \\
Cho/Cr & ns & ns & ns & ns \\
Glx/Cr & ns & ns & r $=0.45,95 \%$ CI $0.079-0.714$ & ns \\
mIns /NAA & ns & ns & ns & r =0.43, 95\% CI $0.044-0.697$ \\
NAA/Cho & ns & ns & ns & ns \\
Glx/NAA & ns & ns & r $=0.43,95 \%$ CI $0.045-0.698$ & ns \\
\hline
\end{tabular}

$\mathrm{DD}=$ disease duration, MSSS $=$ Multiple Sclerosis Severity Scale, $3 \mathrm{VW}=$ width of the third ventricle, $\mathrm{Cho} / \mathrm{Cr}=$ choline to creatine ratio, NAA $/$ $\mathrm{Cr}=\mathrm{N}$-acetyl-aspartate to creatine ratio, $\mathrm{mIns} / \mathrm{NAA}=$ myo-inositol to $\mathrm{N}$-acetyl-aspartate ratio, $\mathrm{Glx} / \mathrm{Cr}=$ glutamate and glutamine to creatine ratio, $\mathrm{Glx} / \mathrm{NAA}=$ glutamate and glutamine to $\mathrm{N}$-acetyl-aspartate ratio, $\mathrm{NAA} / \mathrm{Cho}=\mathrm{N}$-acetyl-aspartate to choline ratio, Pearson correlation coefficient, statistical significance 0.05 .

\section{DISCUSSION}

To our knowledge, this is the first study to date which demonstrates complimentary roles and correlations of subcortical atrophy and hypothalamic glutamate metabolic changes in determining the RRMS disease burden.

Differences between RRMS patients and healthy controls

Significantly decreased NAA/Cr and $\mathrm{Cho} / \mathrm{Cr}$ ratios in RRMS compared to CON are in accordance with our previous study ${ }^{27}$ in which we analysed HYP ${ }^{1} \mathrm{H}-\mathrm{MRS}$ metabolites in a partially overlapping group of patients and controls. In the presented study the patient group was more homogenous - no secondary progressive patients were included. Therefore, concordant results support the existence of HYP neuroaxonal damage (lower NAA) with absence of active demyelination (lower Cho), paralleled by gliosis (higher $\mathrm{Cr}$ ) since the relapse-remitting stage of the disease.

The increase of Glx/NAA in our RRMS was not statistically significant at the $5 \%$ level. This, compared to our previous study with RRMS and secondary progressive (SP) patients ${ }^{27}$ can point to lower degree of neuroaxonal loss (indicated by lower NAA) and/or generally lower burden of the disease in RRMS compared to SP stage (indicated by higher Glu).

In RRMS patients, mIns/NAA increase compared to Glx/NAA seems to be more pronounced, indicating active gliosis since early disease. In accordance with our findings, early mIns increase preceding NAA reduction was reported by Kirov et al. ${ }^{20}$ in pericallosal normal appearing brain tissue.

Differences in $P$-values between this and our previous study ${ }^{27}$ may simply be due to sample size and random error. Further studies with larger cohorts are required to elucidate ${ }^{1} \mathrm{H}-\mathrm{MRS}$ metabolite changes in different MS subgroups compared to normal population.

\section{Predictors of severe disease course}

Logistic regression analysis selected age, 3VW, Glx/ $\mathrm{Cr}, \mathrm{Glx} / \mathrm{NAA}$ as potential predictors of severe disease course in our MS patients, but multiple logistic regression analysis as the relevant predictors identified only $3 \mathrm{VW}$ and Glx/NAA.

\section{Role of glutamate}

In agreement with our previous study ${ }^{27}$ the only ${ }^{1} \mathrm{H}-\mathrm{MRS}$ metabolite parameters correlating with MSSS were those containing Glx, from which G1x/NAA seems to be better than Glx/Cr. Glx/NAA increase, independent from 3VW enlargement, indicates a causal relationship between Glu excitotoxicity, disability and neuronal loss (reflected by NAA), similarly to the findings of Azevedo et al. ${ }^{10}$ analysing supratentorial white and grey matter, and Tisell et al. ${ }^{17}$ analysing perithalamic normal appearing white matter. Our results are the first to demonstrate the usefulness of Glx/NAA in evaluating of infratentorial DGM pathology and suggest a causal relationship between hypothalamic damage and severe course of MS since its relapse-remitting stage.

The questions regarding the precise role of glutamate in MS are still unanswered. Several mechanisms have been hypothesised to be responsible for early alterations in the metabolism of this particular molecule in DGM. One is be based on disturbances of kynurenine, the major degradative pathway of tryptophan, that are associated with Glu toxicity ${ }^{32}$. One of the toxic metabolites - quinolinic acid (QUIN), which facilitates glutamate release by neurons and inhibits glutamate uptake by astrocytes, leads to accumulating Glu in the microenvironment, causing excitotoxicity. QUIN, an agonist of the N-methyl D-aspartate (NMDA) receptor, probably activates the oligodendrocyte-NMDA receptors thus enhancing excitotoxic effect of Glu, subsequent cell death and demyelination ${ }^{32-34}$. Other hypotheses consider mitochondrial dysfunction, defined by reduced mitochondrial genes expression, or decreased activity of inhibitory neurotransmitters such as GABA (ref. $\left.{ }^{35}\right)$.

Epigenetic changes of several neurotransmitters and their receptors are still a matter of debate. For example, over-expression of $\alpha$-amino-3-hydroxy-5-methyl-4isoxazolepropionic acid (AMPA) glutamate receptors was found in the brains of MS patients ${ }^{34}$. Other authors identified genetic variations in brain excitatory neurotransmitters to correlate with neurodegenerative processes in MS $\left(\right.$ ref. $\left.^{36}\right)$. Association of genetic variations of Glu receptors was found to influence MS susceptibility ${ }^{36-38}$. Several authors revealed abnormalities in Glu concentrations due to atypical expression of transporters that regulate its reuptake from the synaptic cleft $^{33,39,40}$. 


\section{The third ventricle width as another relevant predictor of severe disease course}

Value of 3VW planimetry in the assessment of brain atrophy has been historically proven ${ }^{12,21,22}$ and verified by recent studies ${ }^{23,41-43}$. The highly significant difference between RRMS and CON in our work confirms their findings. However, to our best knowledge, in no previous study $3 \mathrm{VW}$ was measured below the hypothalamic sulcus and no study found significant correlations of any planimetric or volumetric parameter of the third ventricle with MSSS $\left(\right.$ ref. $^{44}$ ). The high significance of 3VW-MSSS correlation in our data could result from the good ability of our modification to reflect hypothalamic rather than thalamic atrophy (historically the $3 \mathrm{VW}$ is measured on thalamus level). The hypothalamus was found to degenerate earlier than thalamus in RRMS (ref. ${ }^{3}$ ), however the studies correlating hypothalamic atrophy with clinical outcome of RRMS are absent ${ }^{45}$.

We believe that significant correlations of $3 \mathrm{VW}$ with the independent metabolic and clinical parameters in our study confirm its value in HYP atrophy assessment and validate our modification of the originally proposed method, in which $3 \mathrm{VW}$ was used as a simple general marker of DGM atrophy ${ }^{21}$.

The correlation of $3 \mathrm{VW}$ with MSSS was even more statistically significant than correlation of any metabolic parameter with MSSS. The correlation of 3VW with Glx/ NAA was lower compared to the correlation of $3 \mathrm{VW}$ with mIns/NAA (discussed below), pointing to somewhat independent roles of Glu toxicity versus 3VW in determining the MS severity. We suggest that severe course of the disease, leading to disability before recognisable atrophy, is connected with increased Glu concentrations and thus early diagnosis is possible using ${ }^{1} \mathrm{H}-\mathrm{MRS}$. On the other hand, in moderate RRMS, Glu release may be overlooked (e.g. during a remission period) but the accumulated disability is well reflected by the unavoidable atrophy.

\section{Increased mIns/NAA ratio correlates with the third ventricular width}

We did not identify mIns/NAA as a strong predictor of MSSS, although there was a significant correlation of hypothalamic mIns/NAA with 3VW.

Several other ${ }^{1} \mathrm{H}-\mathrm{MRS}$ studies reported that both the reduction of NAA and increase of mIns positively correlate with disability in MS and can predict its development $\mathrm{t}^{11,19}$, but this correlation was found significant only in the supratentorial white matter and did not reflect severity of MS. In agreement with our results, virtually all published studies failed to prove the correlation of grey matter mIns, NAA or their ratio with MSSS, despite revealing their correlations with the brain atrophy ${ }^{11,17,46}$.

Based on the previously postulated hypothesis of early gliosis preceding neuronal loss ${ }^{20}$, we suggest that glial response may be initially neuroprotective by inhibiting cell-death pathways via triggering neurotrophic factor expression. Later, or in more severe MS, excessive stimulation of a limited amount of glia can lead to dysbalances, causing permanent glutamatergic NMDA activation with neurotoxic effects ${ }^{35}$. Thus, neuronal NAA component can be proportional to DGM atrophy and stable glial mIns component does not disrupt this dependency in the mIns/ NAA ratio, but this ratio is not able to reflect the speed of neurodegeneration due to limited capacity.

\section{Limitations of the study}

Due to the pilot and cross-sectional character of this study, it was conducted at a single site using a single $1.5 \mathrm{~T}$ MRI scanner in a limited cohort of patients and controls. Thus, our findings cannot be fully generalised.

Hypothalamic spectroscopy requires special equipment which is often not available in routine clinical practice. In both patients and controls group we needed to exclude several subjects due to low ${ }^{1} \mathrm{H}-\mathrm{MRS}$ signal. However, the relative number of excluded subjects was low and can be improved by MRI technician training.

No sophisticated approach enabling absolute quantification of metabolites was used and therefore the interpretations of combined ratios may be less straightforward compared to evaluation of single metabolites. On the other hand, many other limitations, inherent to sophisticated absolute quantification procedures, were avoided. Moreover, previous studies showed good informative value of "relative" ratios ${ }^{9-11}$.

The used planimetric measurements are much easier to perform in routine clinical MRI data than hypothalamic volumetry and we tried to maximise the hypothalamus-related information of $3 \mathrm{VW}$ by measuring its value between ${ }^{1} \mathrm{H}-\mathrm{MRS}$ hypothalamic voxels.

\section{CONCLUSION}

Our results suggest that the HYP is a good target structure for evaluation of ${ }^{1} \mathrm{H}$-MRS changes in DGM. Reduction of neuroaxonal metabolic markers (NAA/Cr, $\mathrm{Cho} / \mathrm{Cr}$ ) indicates significant HYP damage in RRMS. Glutamate-related parameters, together with $3 \mathrm{VW}$, were found to be the best in predicting disease burden. Our findings emphasise an important role of neurodegeneration in pathogenesis in RRMS, supporting the excitotoxic hypothesis. Increased mIns ratio parallels atrophy (3VW) and thus points to replacement of hypothalamic neurons by cumulated glia.

Acknowledgement: We thank all the participants for their contribution. This work was supported by Project APVV14-0088/2014, APVV-15-0107, and VEGA 1/0287/16.

Author contributions: HP, EKa: study design and manuscript writing; EKa: clinical tests, MSSS assessment; PH, HP: performed 1H-MRS and 3VW measures, data analysis; MG: statistical analyses; KZ, EKu: critical reading and review of the manuscript.

Conflict of interest statement: The authors state that there are no conflicts of interest regarding the publication of this article.

Ethical statement: This study was performed in accordance with the ethical standards laid down in the 1964 Declaration of Helsinki and its later amendments. Ethics approval was performed by Ethical committee of 
the Jessenius Faculty of Medicine in Martin, Comenius University (EK 1678/2015).

\section{REFERENCES}

1. Miller DH. Biomarkers and surrogate outcomes in neurodegenerative disease: lessons from multiple sclerosis. Neuro Rx 2004;1(2):28494.

2. Lassmann $\mathrm{H}$, BrückW, Lucchinetti C. Heterogeneity of multiple sclerosis pathogenesis: implications for diagnosis and therapy. Trends Mol Med 2001;7:115-21.

3. Haider $L$, Simeonidou $C$, Steinberger $G$, Hametner $S$, Grigoriadis N, Deretzi G, Kovacs GG, Kutzelnigg A, Lassmann H, Frischer JM. Multiple sclerosis deep grey matter: the relation between demyelination, neurodegeneration, inflammation and iron. J Neurol Neurosurg Psychiatry 2014;85:1386-95.

4. Huitinga I, De Groot CJ, Van der Valk P, Kamphorst W, Tilders FJ Swaab DF. Hypothalamic lesions in multiple sclerosis. J Neuropathol Exp Neurol 2001;60:1208-18.

5. Pirko I, Lucchinetti CF, Sriram S, Bakshi R. Gray matter involvement in multiple sclerosis. Neurology 2007;68:634-42.

6. Neema M, Arora A, Healy BC, Guss ZD, Brass SD, Duan Y, Buckle GJ, Glanz BI, Stazzone L, Khoury SJ, Weiner HL, Guttmann CR, Bakshi R. Deep gray matter involvement on brain MRI scans is associated with clinical progression in multiple sclerosis. J Neuroimaging 2009;19:38.

7. Wolinsky JS, Narayana PA, Fenstermacher MJ.Proton magnetic resonance spectroscopy in multiple sclerosis. Neurology 1990;40:1764-9.

8. Helms G, Stawiarz L, Kivisäkk P, Link H. Regression analysis of metabolite concentrations estimated from localized proton MR spectra of active and chronic multiple sclerosis lesions. Magn Reson Med 2000;43:102-10.

9. Caramanos Z, Narayanan S, Arnold DL. 1H-MRS quantification of tNA and $\mathrm{tCr}$ in patients with multiple sclerosis: a meta-analytic review. Brain 2005; 128:2483-506.

10. Azevedo CJ, Kornak J, Chu P, Sampat M, Okuda DT, Cree BA, Nelson SJ, Hauser SL, Pelletier D. In vivo evidence of glutamate toxicity in multiple sclerosis. Ann Neurol 2014;76:269-78.

11. Llufriu S, Kornak J, Ratiney H, Oh J, Brenneman D, Cree BA, Sampat M Hauser SL, Nelson SJ, Pelletier D. Magnetic resonance spectroscopy markers of disease progression in multiple sclerosis. JAMA Neuro 2014;71:840-7.

12. Cifelli A, Arridge M, Jezzard P, Esiri MM, Palace J, Matthews PM. Thalamic neurodegeneration in multiple sclerosis. Ann Neurol 2002;52:650-3.

13. Clark JF, Doepke A, Filosa JA, Wardle RL, Lu A, Meeker TJ, PyneGeithman GJ.N-acetylaspartate as a reservoir for glutamate. Med Hypotheses 2006;67:506-12.

14. Mattson MP. Glutamate and neurotrophic factors in neuronal plasticity and disease. Ann N Y AcadSci 2008;1144:97-112.

15. Choi DW. Glutamate neurotoxicity and diseases of the nervous system. Neuron 1988;1(8):623-34.

16. Macrez R, Stys PK, Vivien D, Lipton SA, Docagne F. Mechanisms of glutamate toxicity in multiple sclerosis: biomarker and therapeutic opportunities. Lancet Neurol 2016;15(10):1089-102.

17. Tisell A, Leinhard OD, Warntjes JB, Aalto A, SmedbyÖ, Landtblom AM, Lundberg P. Increased concentrations of glutamate and glutamine in normal-appearing white matter of patients with multiple sclerosis and normal MR imaging brain scans. PLoS One 2013;8(4):e61817.

18. Brand A, Richter-Landsberg C, Leibfritz D. Multinuclear NMR studies on the energy metabolism of glial and neuronal cells. Dev Neurosci 1993; 15:289-98.

19. Chard DT, Griffin CM, McLean MA, Kapeller P, Kapoor R, Thompson AJ, Miller DH. Brain metabolite changes in cortical grey and normalappearing white matter in clinically early relapsing-remitting multiple sclerosis. Brain 2002;125:2342-52.

20. Kirov II, Patil V, Babb JS, Rusinek H, Herbert J, Gonen O. MR spectroscopy indicates diffuse multiple sclerosis activity during remission. J Neurol Neurosurg Psychiatry 2009;80(12):1330-6.

21. Simon JH, Jacobs LD, Campion MK, Rudick RA, Cookfair DL, Herndon RM, Richert JR, Salazar AM, Fischer JS, Goodkin DE, Simonian N, Lajaunie M, Miller DE, Wende K, Martens-Davidson A, Kinkel RP,
Munschauer FE 3rd, Brownscheidle CM. A longitudinal study of brain atrophy in relapsing multiple sclerosis.The Multiple Sclerosis Collaborative Research Group (MSCRG). Neurology 1999;53:139-48.

22. Benedict RH, Weinstock-Guttman B, Fishman I, Sharma J, Tjoa CW, Bakshi R. Prediction of neuropsychological impairment in multiple sclerosis: comparison of conventional magnetic resonance imaging measures of atrophy and lesion burden. Arch Neurol 2004;61:226-30.

23. Martola J, Stawiarz L, Fredrikson S, Hillert J, Bergstrom J, Flodmark O, Aspelin P, KristoffersenWiberg M. Rate of ventricular enlargement in multiple sclerosis: a nine-year magnetic resonance imaging followup study. Acta Radiol 2008;49:570-9.

24. Polman $\mathrm{CH}$, Reingold SC, Edan G, Filippi M, Hartung HP, Kappos L, Lublin FD, Metz LM, McFarland HF, O'Connor PW, SandbergWollheim M, Thompson AJ, Weinshenker BG, Wolinsky JS. Diagnostic criteria for multiple sclerosis: 2005 revisions to the "McDonald Criteria". Ann Neurol 2005;58:840-6.

25. Roxburgh RH, Seaman SR, Masterman T, Hensiek AE, Sawcer SJ, Vukusic S, Achiti I, Confavreux C, Coustans M, le Page E, Edan G, McDonnell GV, Hawkins S, Trojano M, Liguori M, Cocco E, Marrosu MG, Tesser F, Leone MA, Weber A, Zipp F, Miterski B, Epplen JT, Oturai A, Sørensen PS, Celius EG, Lara NT, Montalban X, Villoslada P, Silva AM, Marta M, Leite I, Dubois B, Rubio J, Butzkueven H, Kilpatrick T, Mycko MP, Selmaj KW, Rio ME, Sá M, Salemi G, Savettieri G, Hillert J, Compston DA. Multiple Sclerosis Severity Score: using disability and disease duration to rate disease severity. Neurology 2005;64:114451.

26. Jiru F, Skoch A, Wagnerova D, Dezortova M, Hajek M. jSIPRO - analysis tool for magnetic resonance spectroscopic imaging.Comput Methods Programs Biomed 2013;112:173-88.

27. Kantorová $E$, Poláček $H$, Bittšanský $M$, Baranovičová $E$, Hnilicová $P$, Čierny D, Sivák Š, Nosál' V, Zeleňák K, Kurča E. Hypothalamic damage in multiple sclerosis correlates with disease activity, disability, depression, and fatigue. Neurol Res 2017;39(4):323-30.

28. R Core Team. R: A language and environment for statistical computing. R Foundation for Statistical Computing. Vienna, Austria. 2015. https://www.R-project.org/.

29. Fletcher TD. Psychometric: Applied Psychometric Theory. R package version 2. 2. 2010. https://CRAN.R-project.org/ package $=$ psychometric

30. Diedenhofen B, Musch J. cocor: a comprehensive solution for the statistical comparison of correlations. PLoS One 2015;10:e0121945.

31. Venables WN, Ripley BD. Modern Applied Statistics with S. 4th edition, Springer. New York. 2002.

32. Lim CK, Brew BJ, Sundaram G, Guillemin GJ.Understanding the roles of the kynurenine pathway in multiple sclerosis progression. Int J Tryptophan Res 2010;3:157-67.

33. Werner P, Pitt D, Raine CS. Multiple sclerosis: altered glutamate homeostasis in lesions correlates with oligodendrocyte and axonal damage. Ann Neurol 2001;50:169-80.

34. Pitt D, Nagelmeier IE, Wilson HC, Raine CS. Glutamate uptake by oligodendrocytes: Implications for excitotoxicity in multiple sclerosis. Neurology 2003;61:1113-20.

35. Dutta R, McDonough J, Yin X, Peterson J, Chang A, Torres T, Gudz T, Macklin WB, Lewis DA, Fox RJ, Rudick R, Mirnics K, Trapp BD. Mitochondrial dysfunction as a cause of axonal degeneration in multiple sclerosis patients. Ann Neurol 2006;59:478-89.

36. Baranzini SE, Srinivasan R, Khankhanian P, Okuda DT, Nelson SJ, Matthews PM, Hauser SL, Oksenberg JR, Pelletier D. Genetic variation influences glutamate concentrations in brains of patients with multiple sclerosis. Brain 2010;133:2603-11.

37. Geurts JJ, Wolswijk G, Bö L, van der Valk P, Polman CH, Troost D, Aronica E. Altered expression patterns of group I and II metabotropic glutamate receptors in multiple sclerosis. Brain 2003;126:1755-66.

38. Rossi S, Studer V, Moscatelli A, Motta C, Coghe G, Fenu G, Caillier S, Buttari F, Mori F, Barbieri F, Castelli M, De Chiara V, Monteleone F, Mancino R, Bernardi G, Baranzini SE, Marrosu MG, Oksenberg JR, Centonze D. Opposite roles of NMDA receptors in relapsing and primary progressive multiple sclerosis. PLoS One 2013;8(6):e67357.

39. Zhou Y, Danbolt NC. GABA and Glutamate Transporters in Brain Front Endocrinol (Lausanne) 2013;4:165.

40. Hardingham GE, Bading $\mathrm{H}$. The Yin and Yang of NMDA receptor signalling. Trends Neurosci 2003;26:81-9.

41. Müller M, Esser R, Kötter K, Voss J, Müller A, Stellmes P. Third ventricular enlargement in early stages of multiple sclerosis is a predictor of 
motor and neuropsychological deficits: a cross-sectional study. BMJ Open 2013;3(9):e003582.

42. Butzkueven H, Kolbe SC, Jolley DJ, Brown JY, Cook MJ, van der Mei IA, Groom PS, Carey J, Eckholdt J, Rubio JP, Taylor BV, Mitchell PJ, Egan GF, Kilpatrick TJ. Validation of linear cerebral atrophy markers in multiple sclerosis. J Clin Neurosci 2008;15:130-7.

43. Goodin DS, Traboulsee A, Knappertz V, Reder AT, Li D, Langdon D, Wolf C, Beckmann K, Konieczny A, Ebers GC; 16-Year Long Term Follow-up Study Investigators. Relationship between early clinical characteristics and long term disability outcomes: 16 year cohort study (follow-up) of the pivotal interferon $\beta-1 \mathrm{~b}$ trial in multiple sclerosis. J Neurol Neurosurg Psychiatry 2012;83:282-7.
44. Shiee N, Bazin PL, Zackowski KM, Farrell SK, Harrison DM, Newsome SD, Ratchford JN, Caffo BS, Calabresi PA, Pham DL, Reich DS. Revisiting brain atrophy and its relationship to disability in multiple sclerosis. PLoS One 2012;7(5):e37049.

45. Lansley J, Mataix-Cols D, Grau M, Radua J, Sastre-Garriga J. Localized grey matter atrophy in multiple sclerosis: a meta-analysis of voxelbased morphometry studies and associations with functional disability. Neurosci Biobehav Rev 2013;37(5):819-30.

46. Geurts JJ, Reuling IE, Vrenken $\mathrm{H}$, Uitdehaag BM, Polman $\mathrm{CH}$, Castelijns JA, Barkhof F, Pouwels PJ. MR spectroscopic evidence for thalamic and hippocampal, but not cortical, damage in multiple sclerosis. Magn Reson Med 2006;55:478-83. 